\title{
Topological Optimization of Compliant Adaptive Wing Structure
}

\author{
M. Santer* \\ Imperial College of Science, Technology, and Medicine, \\ London, England SW7 2AZ, United Kingdom \\ and \\ S. Pellegrino \\ California Institute of Technology, Pasadena, California 91125 \\ DOI: $\underline{10.2514 / 1.36679}$
}

\begin{abstract}
Load-path-based topology optimization is used to synthesize a compliant adaptive aircraft wing leading edge, which deforms in a prescribed way when subject to a single point internal actuation. The load-path-based optimization method requires the specification of a parent lattice. Increasing the complexity of this lattice means the number of parameters required for a complete representation of the structure in the topology optimization becomes prohibitive, although it is desirable to enable a full exploration of the design space. A new method based on graph theory and network analysis is proposed, which enables a substantial reduction in the required number of parameters to represent the parent lattice. The results from this load-path-based approach are compared with those obtained from the better-known density-based topology optimization method.
\end{abstract}

\section{Introduction}

$\mathbf{I}^{\mathrm{N}}$ N RECENT years interest in adaptive aircraft structural concepts has been reawakened, and worldwide there are currently a number of research efforts attempting to realize these concepts in fully functional aircraft. The development of techniques to enable this process is therefore required.

This paper is concerned with the use of computational topological optimization techniques in the design of compliant adaptive aircraft structures. In particular, a concept that enables the wing profile to be changed without the use of conventional hinged flaps will be considered. The approach is to replace the wing rib structure at either or both of the wing leading and trailing edges with a compliant structure, incorporating both the rib and the wing skin, which deforms in a prescribed way when it is subjected to a specified internal actuation. This results in a deformed profile that is considerably more aerodynamically efficient than current flap concepts [1]. A wing concept with a compliant trailing edge has been developed and patented by FlexSys Inc. [2]. In this paper a similar concept is adopted for the design of a compliant rib leading edge, as shown in Fig. 1.

Compliant structures replicate the functionality of conventional mechanisms by means of elastic and, hence, repeatable deformation of the structure itself [3]. There are many advantages to using compliant structures instead of conventional mechanisms. Compliant structures may be designed as single monolithic entities and may therefore be fabricated in a single process. This can result in significant savings, both in cost and time, in the manufacturing process. The absence of relative motion between parts during deformation, such as would occur in a mechanical hinge, is also advantageous, particularly in environments where lubrication may be difficult or unsuitable. Similarly, maintenance requirements may

Received 15 January 2008; revision received 4 November 2008; accepted for publication 9 November 2008. Copyright (C) 2008 by the American Institute of Aeronautics and Astronautics, Inc. All rights reserved. Copies of this paper may be made for personal or internal use, on condition that the copier pay the $\$ 10.00$ per-copy fee to the Copyright Clearance Center, Inc., 222 Rosewood Drive, Danvers, MA 01923; include the code 0001-1452/09 $\$ 10.00$ in correspondence with the CCC.

*Lecturer in Aerostructures, Department of Aeronautics, Prince Consort Road; m.santer@imperial.ac.uk. Member AIAA.

${ }^{\dagger}$ Professor of Aeronautics and Civil Engineering, Graduate Aerospace Laboratories, 1200 East California Boulevard, Mail Code 301-46; sergiop@ caltech.edu. Fellow AIAA. also be reduced. Of particular interest from an aerospace perspective is that the weight and cost of a compliant structure may be considerably lower than the equivalent conventional mechanism. Localized flexure is achieved by means of living hinges, which are thinner regions of the structure whose low bending stiffness facilitates the formation of sharp bends.

One the main difficulties concerning the use of compliant structures is that they need to be designed carefully to achieve the desired shape changes without permanent deformation or fatigue failure of the material. Currently there is a lack of suitable design tools.

This is particularly the case for a structure with distributed compliance, in which changes of configuration result from flexure of the entire structure. This can result in a more efficient use of material than in a structure with localized compliance (in which flexure occurs locally, for example at living hinges), but the behavior of such structures is more difficult to ascertain.

Finding an optimum compliant solution to a particular problem requires techniques that enable the rapid assessment of a large number of different designs. For this reason computational topology optimization schemes are adopted. The primary optimization technique that will be used follows the load-path technique proposed by $\mathrm{Lu}$ and Kota [4] and requires that an initial lattice-structure topology be defined prior at the outset. A genetic algorithm is then used to determine which members should be present in the final optimum topology, which will be a subset of the originally defined topology. The genetic algorithm may also be used to vary the geometry of the members in the initial lattice. A major contribution of the present paper is a method for the reduced parametrization of the topology optimization problem, which enables complex topologies to be synthesized. The load-path technique has yet to become well known, hence it is of interest to compare its results to those from a more standard topology optimization technique, the density-based approach that uses gradient-based algorithms.

In order for topology optimization techniques to become more widely adopted, it is desirable that nonproprietary implementations be available. For this reason, commercial finite-element and optimization software has been adopted. The finite-element software used for the analysis is the commercial software SAMCEF [5], which incorporates a fully nonlinear solver. Both gradient-based and genetic optimization algorithms are integrated with SAMCEF by means of BOSS-quattro [6], which permits parametric studies and optimization to be carried out on fully parameterized finite-element 


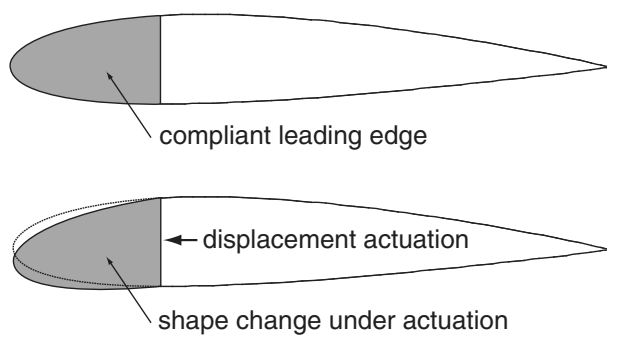

Fig. 1 Compliant adaptive wing leading-edge concept.

models. The density-based continuum optimization approach is implemented in the TOPOL software [7]. The load-path-based optimization approach has been implemented by the authors using existing capabilities within the SAMCEF command language.

Following this introductory section, a detailed description of the load-path topology optimization technique is provided in the next section. It is noted that even a small increase in the number of connecting members in the initial topology rapidly increases the number of parameters required to fully represent the system. In Sec. III a general method using network analysis is proposed that enables the level of parametrization to be systematically reduced while permitting the use of complex initial topologies. Additionally, a method to ensure the manufacturing feasibility of structures optimized with the load-path technique is proposed.

In Sec. IV the optimization problem for the adaptive wing leading edge is defined. The implementation of the optimization problem using the modified load-path technique is then described in Sec. V. The resulting optimized structure is postprocessed, and a functional physical model is fabricated. Section VI assesses the effectiveness of the load-path parameter reduction technique by a comparison with the density-based approach for the same wing leading-edge optimization problem. Finally, a discussion of the results is presented and conclusions are drawn.

\section{Load-Path Topology Optimization}

A general description of the topological and geometrical optimization of a structure with the load-path technique is shown in Fig. 2. This is the starting point for the design process $[4,8]$. The design space $\Omega$ is the region within which the optimized structure must be constrained. For a two-dimensional problem the design space may be defined by means of bounding curves; for threedimensional problems surfaces are used.

On the boundary of the design space lie the input points, output points, and boundary points, which are used to further constrain the structures that are generated by the optimization algorithm. These are the points at which external loads and displacements are defined, the points at which there is a desired output, and the points at which the degree of connection to the ground may be defined, respectively. Within the design space are control points, which are used to define locations through which the structure must pass.

The optimization objective is specified in terms of the minimization of some specified functions, and further constraints,

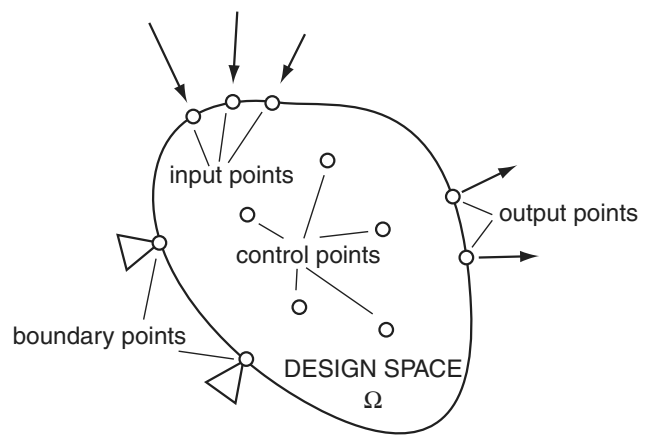

Fig. 2 Generic problem definition for structural optimization. such as the maximum permissible stress, may be specified. The selection of both objectives and constraints is specific to the problem that is being considered and the topology optimization implementation that is used.

In the load-path method an initial structural network of given topology is specified and an optimal structure is sought, whose topology is a subset of the original $[4,8]$. This means that the general structural form is already specified at the beginning of the design process, and therefore minimal postprocessing is required to realize a structure designed by this method, in contrast to the density-based approach, where the material is uniformly distributed at the beginning. The load-path method also enables the inclusion of complex structural elements that would not be possible using a density-based approach. For example, the optimization algorithm can vary the connection type between adjacent members of the lattice between rigid and perfectly hinged. A structure containing localized hinges may then be used as a pseudo rigid-body model of a compliant structure [3].

There are, however, some disadvantages to this approach. For example, all generated structures are dependent on the initially defined parent structure, and therefore the quality of the solution is dependent on the suitability of this initial choice. In addition, if parent topologies with a large number of components are used, which is a requirement if the design space is not well understood, the number of parameters required to define the optimization can become prohibitively large. Methods to reduce the required parametrization level are therefore desirable.

Having defined a parent structure consisting of a lattice of beam members, it is necessary to parameterize this structure to enable the optimization algorithm to remove elements in order to derive new topologies. An intuitive approach would be to use binary parameters to define the existence of each member where one corresponds to a member that exists and zero to a member that does not exist [8]. This method of parametrization, however, leads to significant problems when it is combined with genetic search algorithms. Genetic algorithms (GA) are among the best tools for searching for optimal solutions when the design space is not well understood, as is usually the case for topological optimization $[9,10]$.

With the member parametrization scheme outlined previously, GA iterations are very likely to generate disconnected structures over the course of the optimization; for example, there may be no load path between an input point and the rest of the structure. This may cause the optimization to fail.

A solution to this problem is to adopt an alternative parametrization scheme that assigns a binary parameter, not to individual members, but to sequences of members forming complete load paths [4]. It can be shown that a connected structure will be generated provided there is at least one load path between each input and each output, each output and the ground, and each input and the ground. This is the topology parametrization scheme that is adopted in this paper. However, the automatic generation of load paths for a given parent lattice is by no means straightforward and is discussed in Sec. III.

The final topology determined by the GA is dependent only on the load-path parametrization. However, the GA may be used simultaneously to determine also the optimum geometry of the lattice structure, by introducing a suitably defined additional parametrization. This may include, for example, the beam member cross sections and the control point location within the design space.

\section{Implementation and Extension of Load-Path Technique}

The aim of this section is to present a systematic method for determining the load paths in a parent lattice, to enable a load-path topology optimization to be carried out.

It is useful to illustrate the effects of increasing the complexity of the parent lattice on the number of possible load paths between two points. Figure 3 shows three lattice structures, each having the same unit cell. In all cases, we are interested in the number of possible load paths between the start node (shown shaded) and the end node 


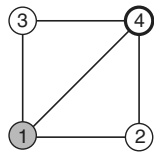

a)

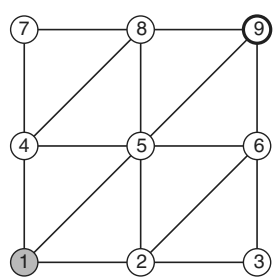

b)

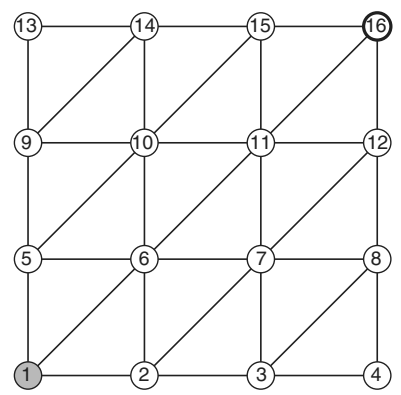

c)

Fig. 3 Lattices of increasing complexity.

(shown with a thick border). It is worthwhile to define exactly what is meant by a load path in this context. A load path is a direct route by which force may be transferred between two chosen nodes in a structure. A load path may therefore contain no closed loops and hence the same node must not appear more than once in any path definition.

Figure $3 \mathrm{a}$ is the simplest lattice structure, consisting of 4 nodes and 5 members. It can readily be seen that there are three load paths between node 1 and node 4: [ [ 1 4], [ $\left.\begin{array}{lll}1 & 2 & 4\end{array}\right]$, and [ $\left.\begin{array}{lll}1 & 3 & 4\end{array}\right]$. At this scale of parent lattice, it makes no difference whether members or load paths are chosen to be parameterized. Figure $3 \mathrm{~b}$ contains 9 nodes and 16 members. Although no closed-form mathematical formula exists for the number of load paths through an arbitrary network, using the KSSP ( $K$ shortest simple path) algorithm described in Sec. III.B, it can be shown that there are 25 load paths between node 1 and node 9 . Increasing the number of members approximately 3 times results in a greater than eight-fold increase in the number of parameters required to represent the lattice fully. Finally, Fig. 3c contains 16 nodes and 33 members; the number of load paths between node 1 and node 16 is 317 .

This example has shown that it is not practical to parameterize all the possible load paths for all but the simplest parent lattice structures.

\section{A. Load-Path Selection}

A possible solution to the rapid increase in the number of load paths with the complexity of the lattice is simply to limit the choice of parent structure to simple lattices. This is not a good solution, however, as limiting the complexity of the starting lattice limits the available topologies that may be generated, as only subsets of this initial structure can be considered. To minimize the effect of initial lattice selection, which is a subjective choice made by the designer, it is desirable that more complex parent lattices be chosen to maximize the number of available subtopologies.

There are two issues that must be addressed when a complex initial lattice is chosen. First, it is necessary to determine the number of load paths required to define the lattice, which may be very high. Second, it is necessary to restrict systematically the number of load paths that are parameterized in the analysis.

A possible restriction method is to limit the number of members that may be linked to form a load path [11]. There are many lattices, however, for which this is not a suitable option. For example, in the lattice shown in Fig. $3 \mathrm{c}$ the shortest load path contains 3 members, there are then 12 load paths with 4 members, and 30 load paths with 5 members. Therefore, this technique provides the designer with limited means to set the level of parametrization of the design problem.

The solution proposed in this paper is to parameterize the $K$ shortest load paths, where $K$ is an integer value that may be defined by the designer. In the preceding example, if $K$ is set to 20 , the parameterized load paths would include all the paths with 3 and 4 members, plus 7 load paths with 5 members. The 5-member load paths that are chosen for parametrization are determined by the order in which they are determined by the algorithm used to search for the load paths.

\section{B. $K$ Shortest Simple Paths}

To determine the $K$ shortest load paths, network analysis theory is used. The parent lattice is represented as an undirected graph $G=$ $[E, N]$ in which $E$ is a set of all the edges in the graph (corresponding to the members in the parent lattice) and $N$ is the set of nodes $v_{i}$ linked by the edges in the graph. An undirected graph means that no distinction is made between the edges $\left[v_{n}, v_{m}\right]$ and $\left[v_{m}, v_{n}\right]$. This is the case here, because in a lattice structure this distinction is not needed. Additionally each edge is equally weighted, as only the existence of that particular edge is of interest.

Having represented the parent lattice as a graph, the determination of the $K$ shortest load paths may be expressed as the well-known $K$ shortest path (KSP) problem in network analysis. The solution of the KSP problem was first attempted in 1959 in order to analyze the traffic flow in Detroit, Michigan [12]. KSP algorithms permit paths with loops to be generated, but it has already been mentioned that in the present case the load paths should be loopless. In graph theory terminology this means that the paths must be simple.

The solution of the KSSP problem is significantly more complex than the solution of KSP. The best-known solution to the KSSP problem is Yen's algorithm, for which a number of implementations have been proposed [13-15]. By way of example, it is now shown how an initial iteration of Yen's KSSP algorithm for the lattice shown in Fig. 3b may be used to generate a list of load paths in increasing order of lengths (using the implementation described in [14]).

The graph representing this structure is shown in Fig. 4a. It should be noted that despite the lengths of the lines of the sketch being different, each edge is assigned a unit cost (illustrated by the value in curly brackets). The load-path start point is node 1 and the end point is node 9 . The first step of the algorithm is to determine a single shortest path using the well-known Dijkstra algorithm.

The shortest-path representation used here is $p_{i}=$ $\left[\begin{array}{lllll}v_{s} & \ldots & v_{t} & \vdots & C\end{array}\right]$ in which path $p_{i}$ links nodes $v_{s}$ to $v_{t}$ and has a total weight $C$, equal to the number of members in the load path. For the lattice in Fig. 4a, Dijkstra's algorithm gives

$$
p_{1}=\left[\begin{array}{lllll}
1 & 5 & 9 & \vdots & 2
\end{array}\right]
$$

The next step is to generate subgraphs that enable deviations from the path under consideration to be determined. These are then used to generate additional shortest-path candidates by reference to the original graph. This is done by removing the nodes and all emanating edges associated with the current (and any already-determined) shortest paths from the network, with the exception of the end node. The minimum tree rooted at the end node is then determined. This involves assigning a number to each node that represents the minimum distance from that node to the end node; this number is shown underlined in the figures. This results in the subgraph shown in Fig. 4b.

The penultimate node of the shortest path is then reinserted into the network along with all its emanating edges except the edge present in the shortest path. The minimum tree rooted at the end node is then updated as shown in Fig. 4c. At this point it is possible to determine some additional shortest paths that deviate from the current shortest 


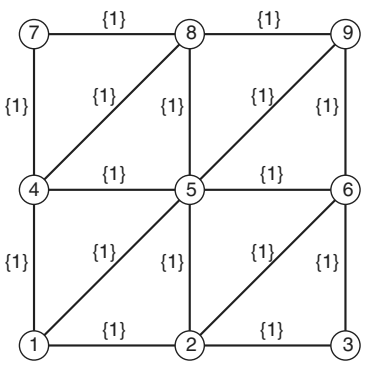

a)

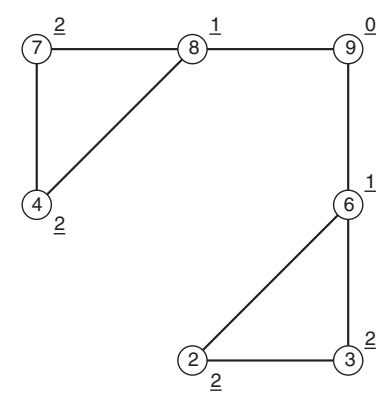

b)

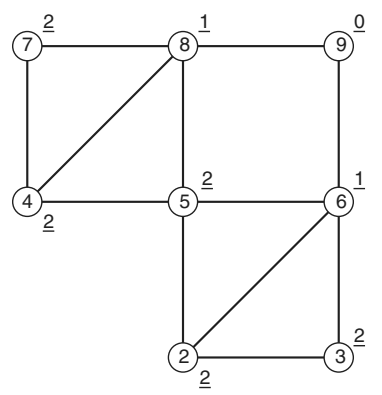

c)

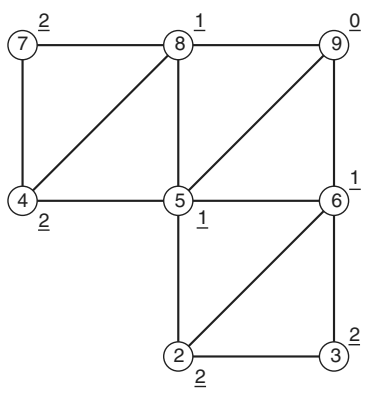

d)

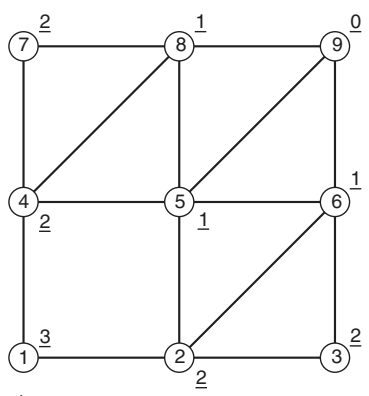

e)

Fig. 4 Illustration of a single iteration of Yen's algorithm.

path at node 5. These deviations are [ $\left.\begin{array}{lll}5 & 6 & 9\end{array}\right]$ and [ $\left.\begin{array}{lll}5 & 8 & 9\end{array}\right]$; they are concatenated with the path $p_{1}$ currently under consideration up to the deviation node to generate additional paths. These paths are stored in a register $[X]$ of candidate shortest paths:

$$
[X]=\left[\begin{array}{llllll}
1 & 5 & 6 & 9 & \vdots & 3 \\
1 & 5 & 8 & 9 & \vdots & 3
\end{array}\right]
$$

The edge emanating from node 5 and contained within the current shortest path is then reinserted in the network and the minimum tree rooted at the end node updated once more, as shown in Fig. 4d.

The next node in the path (in this case the start node) is then reinserted into the network along with all of its emanating edges, except the edge present in the shortest path, and the minimum tree updated in a manner similar to before. The resulting network is shown in Fig. 4e. This enables additional candidate paths to be placed in $[X]$ that deviate from the current shortest path at node 1 as follows:

$$
[X]=\left[\begin{array}{llllll}
1 & 5 & 6 & 9 & \vdots & 3 \\
1 & 5 & 8 & 9 & \vdots & 3 \\
1 & 2 & 5 & 9 & \vdots & 3 \\
1 & 2 & 6 & 9 & \vdots & 3 \\
1 & 4 & 5 & 9 & \vdots & 3 \\
1 & 4 & 8 & 9 & \vdots & 3
\end{array}\right]
$$

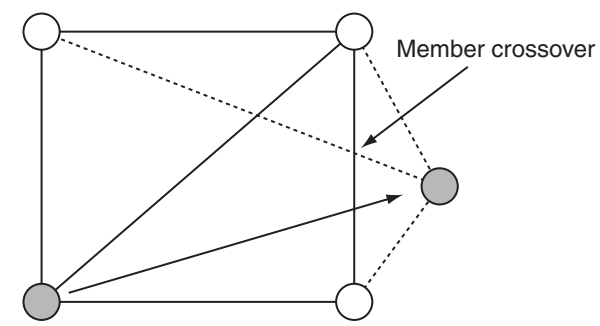

a) Crossover illustration
At this point the next shortest path is selected from the array of candidate paths $[X]$ as the path with the smallest value of $C$. If, however, many candidate paths have the same length [as may be seen in Eq. (3)] then the first candidate path with the shortest length is selected and removed from $[X]$. In this case

$$
p_{2}=\left[\begin{array}{llllll}
1 & 5 & 6 & 9 & \vdots & 3
\end{array}\right]
$$

The process is continued until either $[X]$ is empty or the number of shortest paths found is $K$. It can be seen that this technique provides a systematic method for a designer to control precisely the level of parametrization of the parent lattice and, perhaps equally important, to determine how many load paths are not included in an optimization and consequently how many potential topologies are being neglected.

\section{Feasible Structure Generation}

It is clearly desirable that the optimization process results in an optimum structure that not only satisfies the imposed constraints but is also feasible to construct. Ensuring structural feasibility is, however, often neglected in the implementation of lattice-structure topology optimization.

In particular, if the GA is free to place every control point anywhere within a two-dimensional design space there is a strong likelihood of generating structures with members that cross over each other, and that are therefore not physically realizable. In this paper, a method to prevent member crossover is proposed.

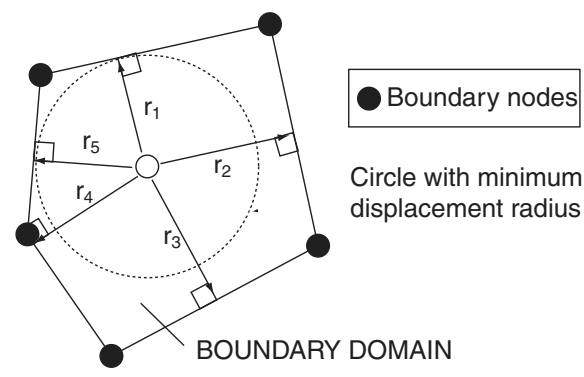

b) Nodal boundary domain

Fig. 5 Member crossover avoidance. 
To a certain extent, the possibility of member crossover can be reduced by careful choice of parent lattices that do not have crossing members. Using this criterion, all the lattices examined in Fig. 3 would be suitable candidates. However, if the control points may be placed anywhere within the design space, such a lattice may still result in unfeasible structures. For example, if in Fig. 5a the shaded node is moved as shown by the optimization algorithm, while all other nodes remain in their original location, a member crossover occurs.

A way of removing the possibility of member crossover is to restrict the movement of control points within the design space. The technique proposed here is to assign all nonfixed nodes a series of boundary nodes that define a polygonal boundary domain, as shown in Fig. 5b.

Each control point is assigned two geometric parameters that determine their displacement, in polar coordinates, from a known datum point. One geometry parameter determines the angle and the other determines the fraction of a maximum permitted radius $r$. This radius is determined from the boundary nodes.

In Fig. $5 \mathrm{~b}$ the control point is shown as a white circle. The boundary nodes, shown as black circles, are defined as the nodes to which there is a direct edge connection (not shown in the figure) from the control point. The boundary nodes form a closed polygon around the control point (the boundary domain). The minimum perpendicular distance $\left(r=\min \left(r_{1}, r_{2}, r_{3}, r_{4}, r_{5}\right)\right)$ from the control point to each of the boundary domain edges is defined as the maximum allowable radius of displacement that the control point may undergo. By restricting the geometric changes of the control point locations in this way, the possibility of member crossover is removed.

\section{Wing Leading-Edge Optimization Problem Definition}

In this section the topology optimization problem will be defined for the compliant adaptive leading edge shown in Fig. 1. The wing is envisaged to be incorporated in an unmanned aerial vehicle (UAV) and the Sky-X UAV developed by Alenia-Aeronautica is taken as the basis for the chosen dimensions and loadings. The wing profile is set as the NACA-2421 profile having a chord length of $1056 \mathrm{~mm}$. The leading-edge portion of the wing (the region that will be populated with adaptive compliant structure) is defined as consisting of the first quarter chord $c / 4=264 \mathrm{~mm}$. The rib spacing is chosen to be $250 \mathrm{~mm}$ and the wing skin thickness is set at $1 \mathrm{~mm}$. Each rib is $5 \mathrm{~mm}$
Table 1 High-strength aluminium material properties

\begin{tabular}{lc}
\hline \hline Young's modulus, $\mathrm{N} / \mathrm{mm}^{2}$ & 72,000 \\
Poisson's ratio & 0.33 \\
Yield strength, $\mathrm{N} / \mathrm{mm}^{2}$ & 395 \\
\hline \hline
\end{tabular}

thick. The material used is high-strength aluminium, having the properties listed in Table 1.

A schematic diagram of three consecutive ribs is shown in Fig. 6. Part of the central rib is highlighted to identify the leading-edge design domain $\Omega$ and the wing skin connected to it. A SAMCEF finite-element model [16] was created of the leading-edge section bounded by the lines and curves $\mathrm{AB}, \mathrm{CE}, \mathrm{DF}, \mathrm{CD}$, and EF. The wing skin was modeled with a uniform mesh of Mindlin shell elements, as shown on the right hand side of Fig. $\underline{6}$. The modelling of the compliant rib structure and the interface between the rib and the skin is described in relation to the topology optimization techniques, in the relevant sections following.

Periodic boundary conditions are applied to curve CE and curve DF to represent the interaction of the section of wing that is modeled with the rest of the structure. These edges therefore have their spanwise ( $z$ axis) displacement restrained but are free to translate in the $x-y$ plane. The model is fully fixed at the interface between the leading edge, which is the design domain of the topology optimization, and the rest of the wing. This interface is defined by the straight lines $\mathrm{CD}$ and EF, as defined in Fig. 6. The adaptive leading edge is required to effect a prescribed shape change when subjected to a combination of aerodynamic pressure loading and internal actuation. To determine the pressure loading, a flight speed of $260 \mathrm{kt}$ $(134 \mathrm{~m} / \mathrm{s})$ at sea level and a $5 \mathrm{deg}$ angle of attack were assumed as representative flight conditions for the UAV. The two-dimensional pressure distribution was evaluated for an inviscid flow condition using the XFOIL software [17]. In the optimization this pressure was mapped onto the wing skin as shown in Fig. 7. It must be noted that this is not an aeroelastic analysis, as no account is taken of the change in pressure loading due to the change in wing profile under actuation, but this approach ensures that the compliant structure generated by the optimization algorithm includes the effects of an aerodynamic loading of the correct order of magnitude.

The actuation to cause the shape change is a fixed chordwise displacement of $10 \mathrm{~mm}$, applied at the midpoint of $\mathrm{AB}$. The combination of actuation and pressure loading shown in Fig. 7 is present in all subsequent design cases.

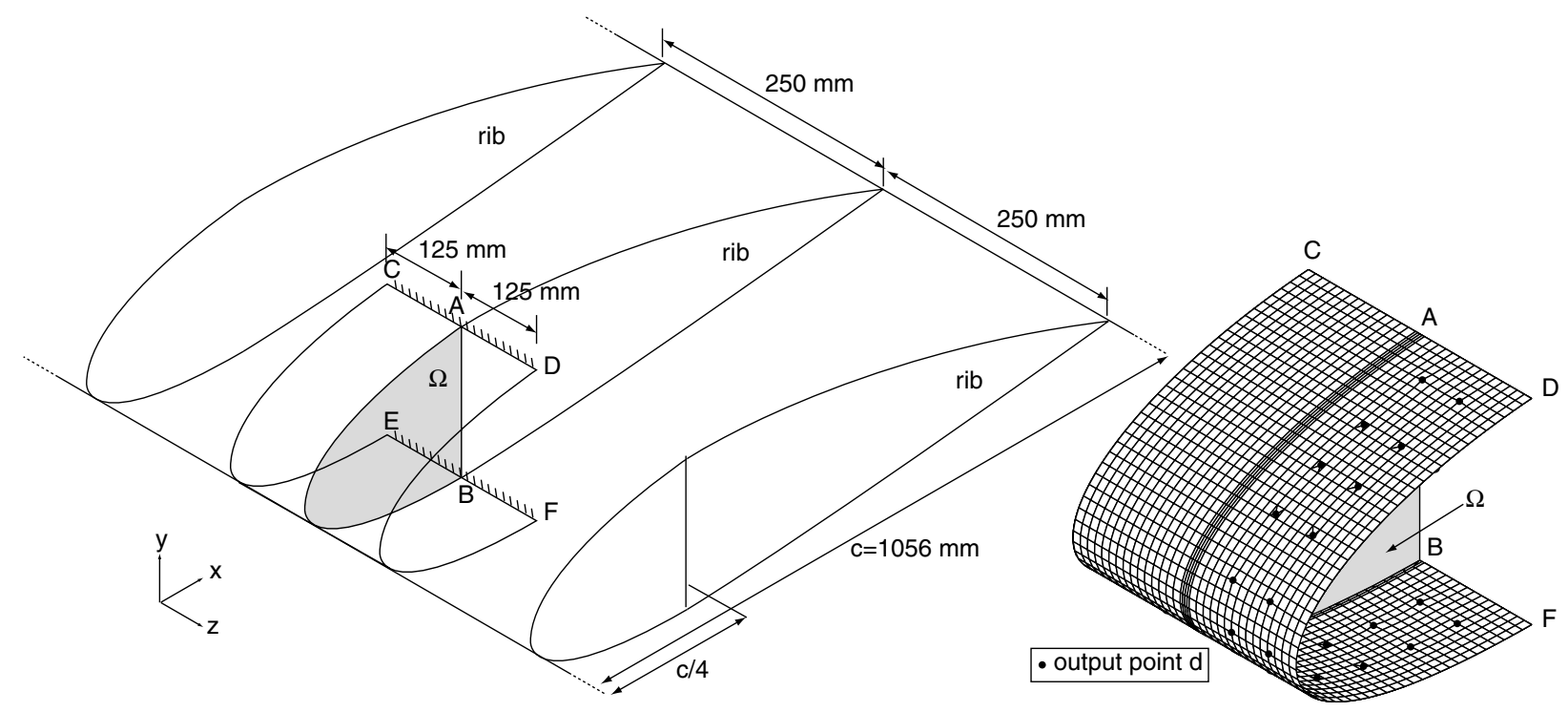




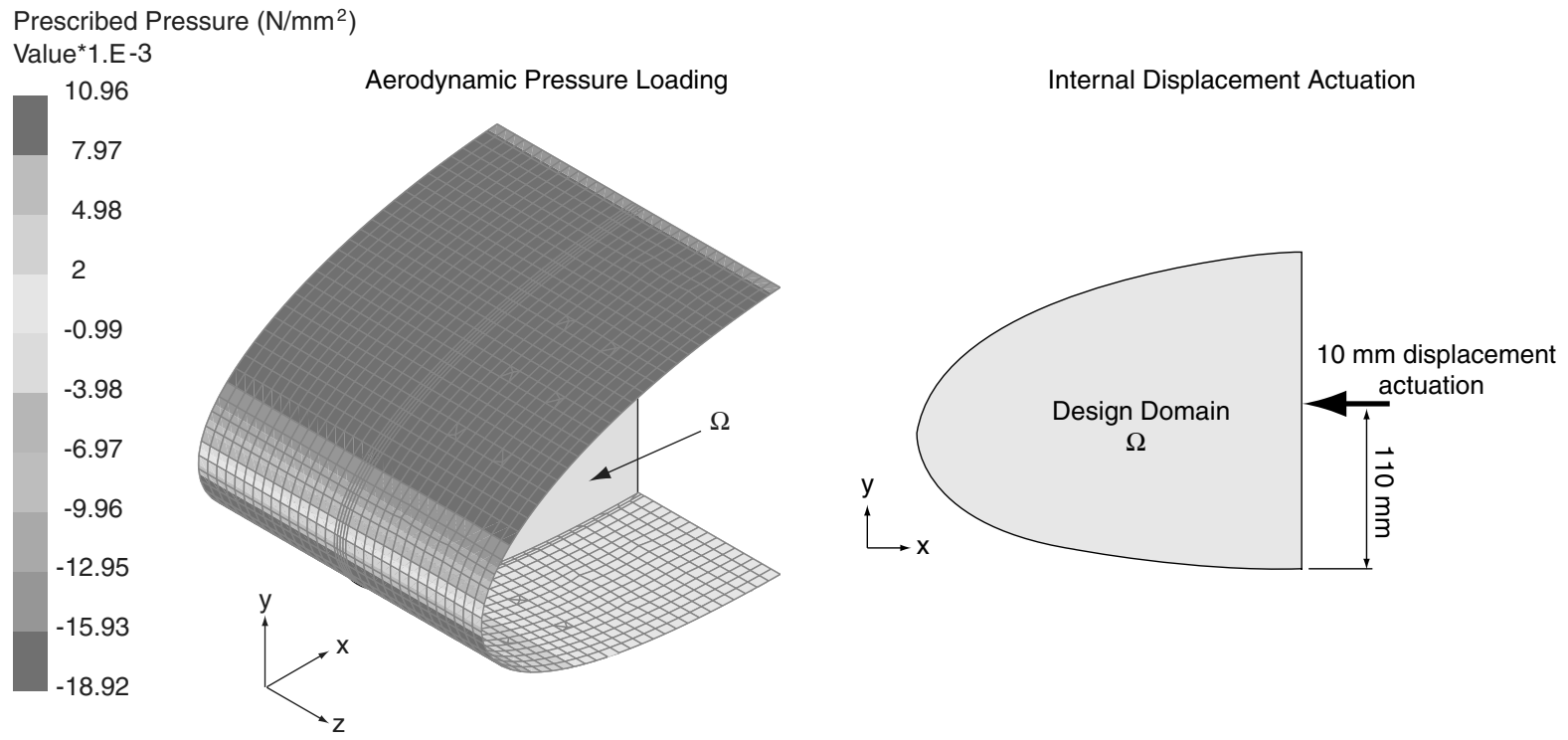

Fig. 7 Aerodynamic pressure loading and internal actuation resulting in leading-edge shape change.

The topology optimization will be mathematically defined in Sec. V.A, but it may be described qualitatively as the following dualobjective problem: determine the structure that provides

$$
\min (\operatorname{mass})
$$

$\min ($ shape change error)

subject to the previously defined loadings and boundary conditions. Additional constraints relevant to the chosen topology optimization technique may also be specified. The shape-change error is defined by reference to 20 output points located in two rows of ten, as indicated in Fig. 6. The vertical ( $y$ axis) displacement is desired to be that obtained by a downward 5 deg rotation of the leading edge about the quarter-chord. The points are approximately equally spaced along the profile, and the two rows are to ensure that the deformation under actuation is approximately two-dimensional (i.e., the difference in vertical deflection at two output points with the same $x$ and $y$-coordinates is negligible). The coordinates of the output points $d_{i}(i=1 \ldots 10)$ and the corresponding desired deflections $\delta_{i}$ are shown in Table 2. For $d_{i+10}$ the values are the same as for $d_{i}$ with the exception that the $z$ coordinate is $83.333 \mathrm{~mm}$.

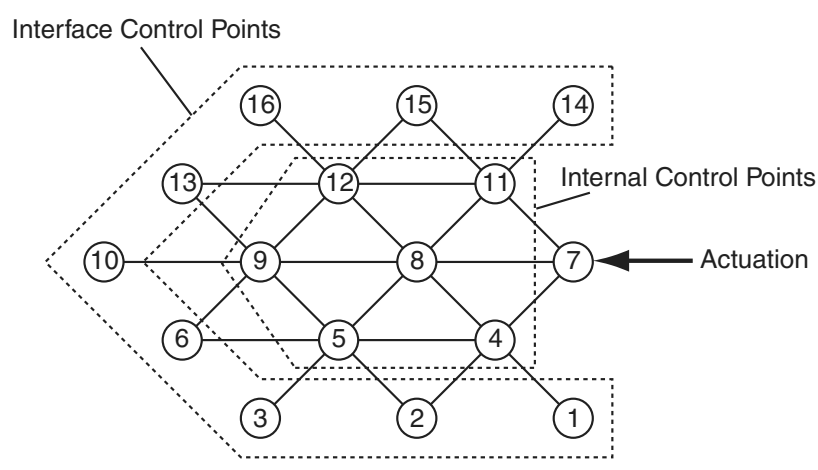

a) Parent lattice graph

\section{Load-Path Optimization}

\section{A. Optimization Problem}

In this section the topology optimization problem will be formulated for use with the load-path-based technique. The first stage is to define a parent lattice within the design domain that forms the basis of all subsequently generated topologies. The chosen lattice, which is a subjective choice of the designer, is shown in Fig. 8a. It should be noted that the structure is free from member crossover to assist with the generation of feasible structures.

The control points, which determine the geometry of the generated structures, are categorized as internal and interface control points. The former are free to move within the design space (subject to anticrossover constraints) whereas the latter are constrained to lie on the boundary, determined by curve $\mathrm{AB}$ in Fig. 8b. The interface control points determine the locations where there is a connection between the rib and the wing skin. This connection is through an additional $5 \mathrm{~mm}$ wide and $0.5 \mathrm{~mm}$ thick strip, modeled with Mindlin shell elements, which may be seen along curve AB in Fig. $\underline{6}$. The control point coordinates are listed in Table 3 .

For the purposes of defining a load-path optimization, it is only necessary to ensure that there is at least one load path between the input (actuation) point and any one of the interface control points. All the remaining required connections are ensured by the continuous presence of the wing skin. This is because the wing skin is fixed at the

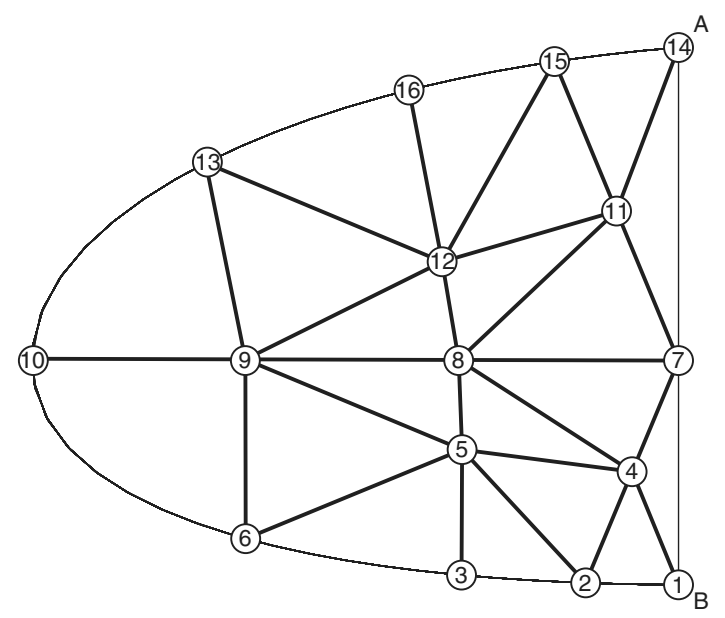

b) Parent lattice realization

Fig. 8 Definition of parent lattice. 
Table 2 Output points to determine shape-change error, including coordinates and desired vertical deflection $\delta$

\begin{tabular}{|c|c|c|c|c|c|c|c|c|c|c|}
\hline & $d_{1}$ & $d_{2}$ & $d_{3}$ & $d_{4}$ & $d_{5}$ & $d_{6}$ & $d_{7}$ & $d_{8}$ & $d_{9}$ & $d_{10}$ \\
\hline$x, \mathrm{~mm}$ & 224.932 & 160.885 & 111.302 & 60.47 & 12.605 & 14.363 & 64.945 & 117.326 & 170.205 & 222.504 \\
\hline$y, \mathrm{~mm}$ & 123.808 & 112.24 & 97.839 & 75.171 & 35.486 & -35.01 & -65.65 & -79.708 & -87.172 & -90.704 \\
\hline$z, \mathrm{~mm}$ & 41.667 & 41.667 & 41.667 & 41.667 & 41.667 & 41.667 & 41.667 & 41.667 & 41.667 & 41.667 \\
\hline$\delta$ & -3.876 & -9.414 & -13.681 & -18.025 & -22.046 & -21.624 & -17.099 & -12.48 & -7.843 & -3.271 \\
\hline
\end{tabular}

Table 3 Starting control point coordinates

\begin{tabular}{|c|c|c|c|c|c|c|c|c|}
\hline Point & 1 & 2 & 3 & 4 & 5 & 6 & 7 & 8 \\
\hline$x$ & 262.283 & 246.481 & 229.405 & 261.221 & 228.536 & 208.829 & 264 & 222.636 \\
\hline$y$ & -91.639 & -91.426 & -90.953 & -40.061 & -40.579 & -90.067 & 7.02 & -24.324 \\
\hline Point & 9 & 10 & 11 & 12 & 13 & 14 & 15 & 16 \\
\hline$x$ & 207.435 & 168.641 & 120.548 & 190.32 & 118.147 & 127.944 & 17.676 & 81.974 \\
\hline$y$ & -52.292 & -87.016 & -16.268 & -34.613 & -79.865 & 103.332 & -38.414 & -71.268 \\
\hline
\end{tabular}

Table 4 Optimized control point coordinates

\begin{tabular}{|c|c|c|c|c|c|c|c|c|}
\hline Point & 1 & 2 & 3 & 4 & 5 & 6 & 7 & 8 \\
\hline$x$ & 264 & 226.088 & $(175.392)$ & 245.044 & 175.627 & 87.254 & 264 & 174.242 \\
\hline$y$ & -91.641 & -90.839 & $(-87.666)$ & -45.42 & -36.386 & -72.772 & 0 & 0.254 \\
\hline Point & 9 & 10 & 11 & 12 & 13 & 14 & 15 & 16 \\
\hline$x$ & 87.124 & 0.005 & 238.692 & 167.469 & 70.937 & 264 & 213.384 & $(153.959)$ \\
\hline$y$ & 0.5 & 0.747 & 61.099 & 40.385 & 80.77 & 127.923 & 122.197 & $(110.56)$ \\
\hline
\end{tabular}

interface with the quarter-chord and the output points, which are used to measure the shape change under actuation, also lie on the skin. Therefore there is always a load path between the output points and the ground and from the interface control points to the output points. The required existence of a single load path may be expressed as a constraint.

To parameterize the structure completely, a total of 578 (evaluated using the KSSP algorithm) load paths should be considered. However, a reduced set was used consisting of the 10 shortest paths between the input and each of the nine output points. Referring to Fig. 8a, this corresponds to 90 load paths, 10 between node 7 and each of nodes $1,2,3,6,10,13,16,15$, and 14 . The placing of the idealized parent lattice onto the wing rib geometry is shown in Fig. 8b. The coordinates $\left(x_{i}, y_{i}\right)$ of the 16 control points are also parameterized. The chromosome used in the GA may be represented as

$$
\left\{\left[\left(x_{i}, y_{i}\right), i=1 \cdots 16, \text { continuous }\right], \quad\left[p_{i}, i=1 \cdots 90, \text { binary }\right]\right\}
$$

Expressing Eqs. (5) and (6) in forms appropriate to the load-pathbased optimization results in the following optimization problem

$$
\begin{array}{ccc}
\min \left(\sum_{i} p_{i}\right) & i=1 \cdots 90 & \text { (mass objective) } \\
\min \left(\sqrt{\frac{\sum_{i}\left(d_{i}-\delta_{i}\right)^{2}}{i}}\right) & i=1 \cdots 20 & \text { (shape change objective) } \\
\text { s.t. } & \text { (connectivity constraint) }
\end{array}
$$

It will be noted that the mass objective is formulated as the sum of the load paths in the particular topology. Strictly this is not the actual mass of the structure, as different members in the lattice have different lengths. Similarly the same member may contribute to more than one load path. The reason for this formulation is that the objective encourages the generation of simple structures, that is, a larger number of load paths corresponds to a more complex topology. Structures with fewer load paths will be lightweight as desired, although not necessarily optimally so.

The objective can be reformulated as $\min \left(W+\sum_{i} p_{i}\right)$, in which $W$ is the total weight of the structure, to ensure an optimally lightweight solution. This was found to hinder convergence in this particular problem, however, and was not adopted. The shapechange error is defined as the root-mean-square (rms) error of the points as specified in Table 2 .

For the load-path-based optimization, the two objective functions in Eq. (8) are combined using a weighted sum approach in which a composite objective function is formulated according to

$$
\min \left(\sum_{i=1}^{2} w_{i} f_{i}\right)
$$

The mass objective is denoted by $f_{1}$ and the shape-change objective by $f_{2}$. Equal weights $w_{i}$ are assigned to both objectives, reflecting their equal importance in the design problem.

\section{B. Genetic Algorithm}

A genetic algorithm is used in the load-path-based topology optimization due to the combination of continuous (control point coordinates) and discrete (load-path existence) parameters, and because the random nature enables a thorough search of the problem space. The GA implementation in the commercial optimization software BOSS-quattro [18] is used. Model parametrization for the implementation of the load-path-based topology optimization was incorporated into the SAMCEF finite-element software [19]. A description of the GA settings and strategies follows.

In each iteration of the GA, all members of the population are evaluated for suitability. There is a crossover probability of 0.9 , representing the chance that two individuals in the population are modified during reproduction. There is a 0.01 mutation probability, which represents the chance that an individual in the population will mutate during its life. An elitism strategy is adopted to ensure that the single best individual in an old population is carried over to the new population if it does not already exist. One-point crossover is used for the creation of two children from two parents. One crossover position 
is selected in the chromosome string. Variables are exchanged between the individuals about this point to produce two new children.

A tournament selection is used to assess the fitness of individuals in the population. In this process, two individuals are chosen at random from the population, the best of which is selected as a parent. This process is repeated for all individuals. To ensure a continued diversity of the population, a rebirth strategy is adopted. Every 10 evolutions, the worst half of the population is replaced by completely new individuals. This ensures that as much of the design space as possible is explored.

\section{Load-Path-Based Optimization Results}

Following 3100 evolutions, the structure that best achieved the objectives shown in Fig. 9a was found to have been generated at iteration 3042. A nonlinear arc-length-based analysis was then carried out to determine the shape change resulting from the displacement actuation. The result is shown in Fig. 9b. It can be seen that there is no local buckling of the members. It should be noted that Fig. $9 \mathrm{~b}$ is an end view of a three-dimensional structure. It can be seen that the deformed shape of the skin, modeled with shell elements, is not completely two-dimensional, as there is a small amount of spanwise variation. The modified coordinates of the 16 control points are listed in Table 4 . Coordinates for points 3 and 16 are evaluated but do not form part of the solution as they are not in a required load path.

The rms error of the shape change is $0.83 \mathrm{~mm}$ and the number of required load paths to attain the structure shown in Fig. 9a is 5. It can be seen that the stress under the combined effects of actuation and aerodynamic pressure loading (which was not constrained in the optimizations), is $1353 \mathrm{MPa}$. This is greater than the material yield stress, shown in Table 1 and must be reduced in a subsequent step if the structure is to be realized.

\section{Postprocessing and Fabrication}

This section illustrates the process of refinement and implementation of the optimized solution shown in Fig. 9a in order to realize a physical demonstration model of a single compliant rib.

The first stage is to remove the aerodynamic loading and the wing skin (leaving the connection strip defined in Sec. V.A). This, in fact, has a limited effect on the peak stress, as the displacement actuation has a significantly greater effect on the stresses than the aerodynamic loading, and the highest stresses are in the rib members. The peak stresses are concentrated in the two members labeled as highly stressed in Fig. 9b. They are reduced in two different ways, as follows.

Member 1 is in tension, and high stresses in this member occur as a result of large bending moments at the ends. The solution is to replace the member connection type at each end with hinges that are implemented as living hinges. These hinges are available as a dedicated element in SAMCEF, which is a multipoint constraint, fixing the relative translations and rotations with the exception of the rotation about the $z$ axis. Member 2 is also subjected to high bending moments but it is in compression. Therefore, placing living hinges at the ends is not a good solution as they would reduce the resistance to buckling. Instead this member is simply removed as this is found to have only a minimal effect on the shape adopted by the rib under actuation. These modifications are shown in Fig. 10a.

The hinges were realized as 0.5 -mm-thick living hinges. All corners were rounded to a radius of at least $1 \mathrm{~mm}$, to avoid stress concentrations. To confirm that the modified model behaved in the desired manner, a finite-element model using solid elements was analyzed. The shape change and von Mises stress of this modified model under actuation is shown in Fig. 10b. The actuation displacement is reduced to $6 \mathrm{~mm}$ as the wing skin is not included in the model. It can be seen that the peak stress in the compliant rib is $303 \mathrm{~N} / \mathrm{mm}^{2}$, which provides a $30 \%$ margin against material yield.

\section{E. Physical Model}

A full-size demonstration model of the design shown in Fig. 10b was constructed. Figure 11a shows the model in its undeformed, unactuated configuration. Figure $11 \mathrm{~b}$ demonstrates the effect of a displacement actuation of $4.8 \mathrm{~mm}(80 \%$ of the actuation shown in Fig. 10b) applied by means of a screw. The undeformed shape is indicated by the black line. The model returns fully to its initial configuration when the actuation is removed. This behavior illustrates the success of the design approach.

\section{Density-Based Optimization}

In the previous section the reduced parametrization of the loadpath topology optimization using KSSP analysis enabled the design of a compliant leading edge that responds with a desired shape change when it is subjected to combined actuation and aerodynamic loading. The outcome was the compliant rib design illustrated in Fig. 9a. The use of load-path-based optimization, in particular with reduced parametrization, is not as well-established as density-based topology optimization techniques. Hence, it is of interest to provide some validation of the achieved topology by comparison to topologies obtained for the same design problem using the densitybased approach. A commercial density-based topology optimization implementation, TOPOL [7] $]$, is used.

\section{A. Density-Based Topology Optimization}

The use of binary material properties leads to an ill-posed optimization problem, and so the material distribution topology optimization routine that is implemented in TOPOL [20] is the density-based approach simple isotropic material with penalization

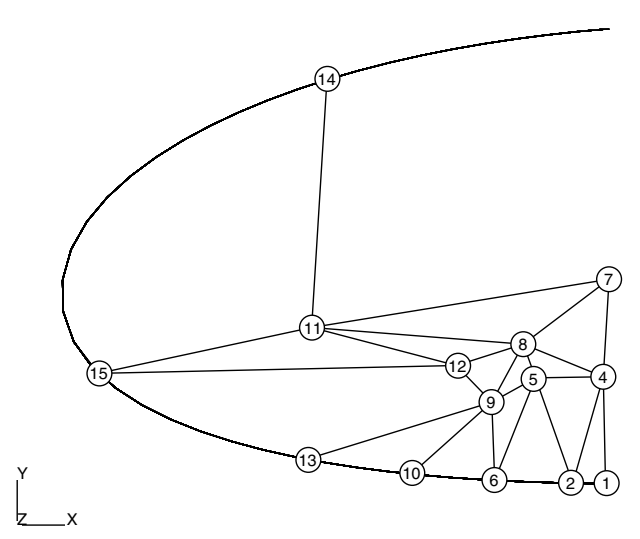

a)

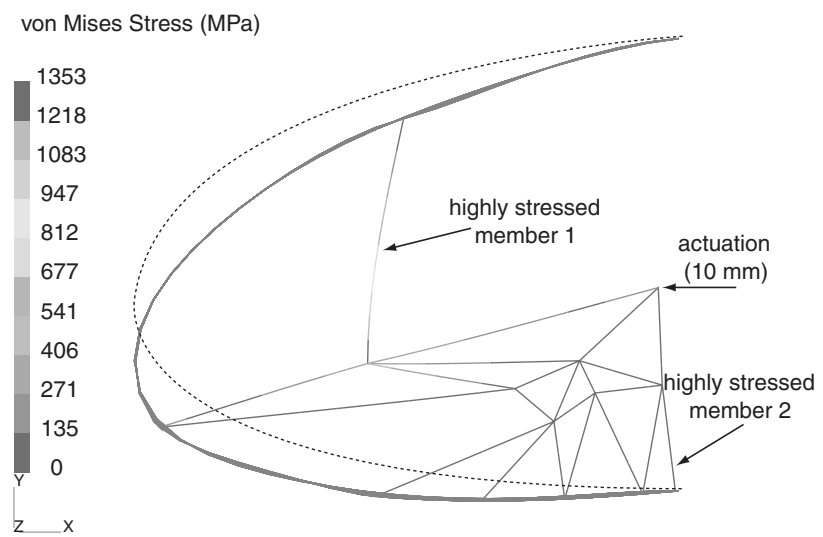

b)

Fig. 9 Optimized compliant leading edge. 


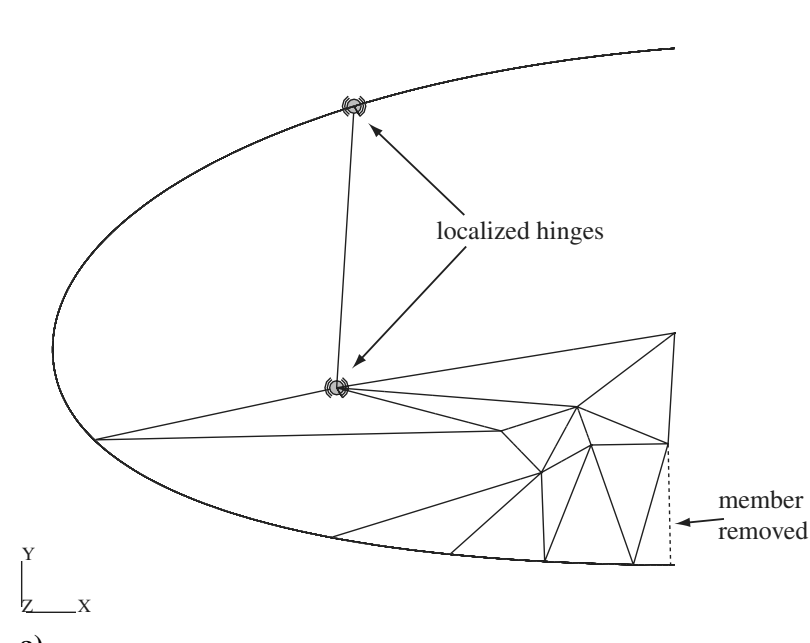

von Mises Stress (MPa)

a)

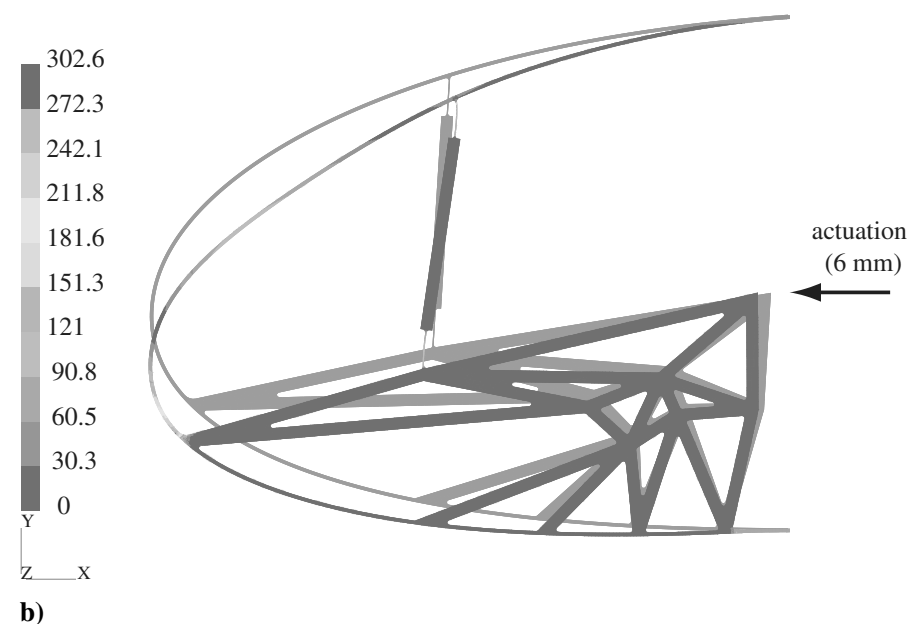

Fig. 10 Manually modified solution for rib only.

(SIMP) [21], which is a good alternative to the more complex homogenization technique. This technique is an artificial material method in which the density of individual elements is varied according to the relationships

$$
\rho=\mu \rho_{0} \quad \text { and } \quad E=\mu^{n} E_{0}
$$

which may be more concisely expressed by the power law [22]

$$
\frac{E}{E_{0}}=\left(\frac{\rho}{\rho_{0}}\right)^{n}
$$

Here, the subscript 0 represents the properties of a given isotropic material. The density $\rho$ is the design variable that varies the material properties between the two extremes of no material at all and the given isotropic material.

In practical implementations, the minimum density is usually constrained to a small percentage of $\rho_{0}$ to prevent singularities. The value of the exponent $n$ is a design variable that may be chosen. Typically, smaller values of $n$ lead to structures with larger members with blurred edges and large material density variations, along with higher values to more slender members with a more binary material distribution. One approach that assists with the avoidance of local minima is the use of a continuation method in which the value of the exponent $n$ is increased as the iteration proceeds [21].

Some modifications to the SIMP approach have been proposed that result in optimized structures without intermediate densities. This includes the incorporation of filter functions, for example the Heaviside step function [23], in the optimization scheme. Many additional morphology-based filter functions are also available [24]. Filter functions may also be implemented to avoid solutions showing checkerboarding (i.e., regions with high density and low density
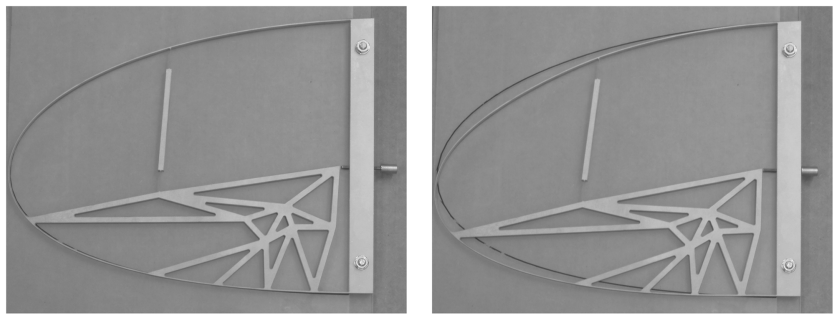

a)

b)

Fig. 11 Two configurations of demonstration model showing a) before actuation, and b) after imposing displacement of $6 \mathbf{~ m m}$. material in close proximity). For example, the Heaviside filter produces checkerboard-free designs. An additional solution is to make the density of a particular element sensitive to those of its immediate neighbors [21]. It is also possible to obtain a black-andwhite structure by means of a postprocessing filter that, for example, removes all elements below a prescribed density and returns all remaining elements to the properties corresponding to the given isotropic material. Although no longer strictly an optimum solution, the resulting structure will be significantly easier to manufacture.

Following a density-based topology optimization it is often necessary to postprocess the solution to make the discrete structure physically realizable; for example, locally flexible regions have to be transformed into living hinges. Techniques exist for carrying this out [25], but significant engineering judgement may be required during this process. This is cited as one of the primary disadvantages of material distribution topology optimization [11]. A strong advantage of the method, however, is that it requires no initial hypothesis about the kind of structural topology that is required; the continuum is simply fully populated with elements at the start of the optimization process.

The optimization algorithm that is implemented in TOPOL is the gradient-based convex-linearization algorithm [26]. This algorithm carries out a linearization of all functions involved in the optimization using either a direct or a reciprocal formulation depending of whether the gradient of the function is positive or negative. This linear updating of the set of functions $f\left(p_{i}\right)$ is carried out according to

$$
f\left(p_{i}\right) \approx f\left(p_{i}^{0}\right)+\overbrace{\sum_{>0} \frac{\mathrm{d} f}{\mathrm{~d} p_{i}^{0}}\left(p_{i}-p_{i}^{0}\right)}^{\text {direct linearization }}+\overbrace{\sum_{<0} \frac{\mathrm{d} f}{\mathrm{~d} p_{i}^{0}}\left(p_{i}^{0}\right)^{2}\left(\frac{1}{p_{i}^{0}}-\frac{1}{p_{i}}\right)}^{\text {reciprocal linearization }}
$$

\section{B. Density-Based Topology Optimization}

To carry out a topology optimization of the adaptive wing leading edge using TOPOL it is necessary to reformulate the optimization problem in an appropriate fashion. In the current version of the software it is only possible to specify the mass minimization as an objective, and it is therefore necessary to reexpress Eq. (6) in terms of constraints. Hence, the minimum allowable density $\rho_{\min }$ is specified as an additional constraint.

The reformulated optimization problem, which may be compared with Eq. (8), is therefore expressed as 


$$
\begin{array}{ccc}
\min \left(\sum_{i} \rho_{i} V_{i}\right) & & \text { (mass objective) } \\
\text { s.t. } & & \\
\delta_{i}-\epsilon / 2<d_{i} & i=1 \cdots 20 & \text { (shape change reexpressed as }<\text { constraints) } \\
\delta_{i}+\epsilon / 2>d_{i} & i=1 \cdots 20 & \text { (shape change reexpressed as }>\text { constraints) } \\
\rho_{\min } \leq \rho_{i} \leq \rho_{0} & & \text { (minimum density constraint) }
\end{array}
$$

The mass objective and the minimum density constraint are evaluated over all the elements in the optimization. The desired shape change corresponds to the value $\delta_{i}$ at output point $i$. However, in order to express the desired shape change in terms of inequalities, it is necessary to introduce an additional parameter $\epsilon$, which specifies an acceptable range for the shape-change error.

The model, material, loading, and boundary conditions are identical to those previously specified for the load-path-based optimization. The design domain $\Omega$ is initially fully populated with a single layer of hexahedral brick finite elements. A SIMP penalty $n=3$ was adopted, which was held constant throughout the iterations. Although a linear continuation of $n$ is available in TOPOL, it was not used. The minimum density $\rho_{\min }$ was set at $5 \%$ of the given material (high-strength aluminium) density.

\section{Density-Based Optimization Results}

An initial observation was that the density-based approach is not well suited to the optimization problem as formulated in Eq. (13). It was observed that, after approximately 20 iterations, the displacement constraints start to compete and oscillatory behavior ensues. It was found that the final solution was strongly dependent on the specification of the shape-change constraints. Some of these solutions, for particular shape-change constraint specification, which gave the best rms shape-change error under actuation and aerodynamic loading, are shown in Fig. 12. They have not been subjected to any postprocessing filtering, as this obscures the achieved topology. They should be compared with the load-pathbased solution shown in Fig. 9a.

In the figure, the value of $\epsilon$ expresses the permissible deviation at the output points from the desired shape change. It can be seen that the best (i.e., most black and white) results are achieved when $\epsilon$ is small. Full constraint means that all 20 output points were defined as constraints in the topology optimization. It is interesting to see the effect on the optimization of reducing the number of output points. Partial constraint in Fig. 12 indicates that the "less than" constraints in Eq. (13) were restricted to output points $d_{i}(i=3,5,7,9$, $13,15,17,19)$, and the "greater than" constraints were applied only to output points $d_{i}(i=6,16)$. In these cases the rms error is calculated based only on the output points that are used. It can be seen that the best rms error that was achieved was $7.7 \mathrm{~mm}$.

When comparing the results to the load-path-based solution in Fig. 9a, two similarities are apparent. The first is that a rigid area is required near the bottom right-hand corner of the rib. The second is that a thin region of structure is required all around the interface between the rib and the structure. In the case of the load-path-based optimization this was specified before the optimization; its presence in the density-based optimization solutions acts as a validation. The optimal topology of the remainder of the structure is difficult to determine from the density-based topology optimization approach, although the presence of a diagonal member connecting the rigid section to the top wing skin is indicated by the solutions shown in Fig. 12b-12d. In all cases the rms shape-change error is significantly higher than that achieved with the load-path-based topology optimization.

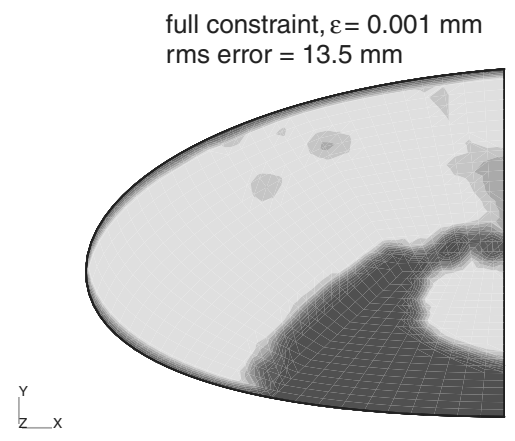

a)

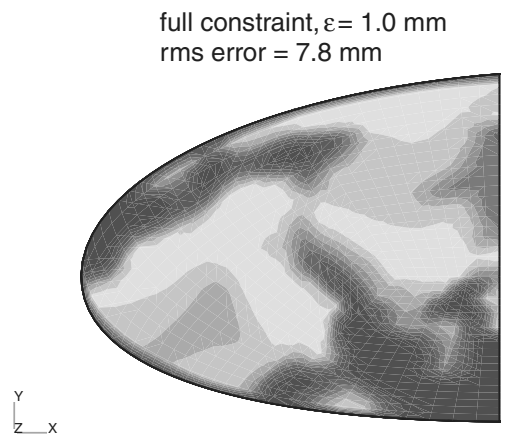

c)

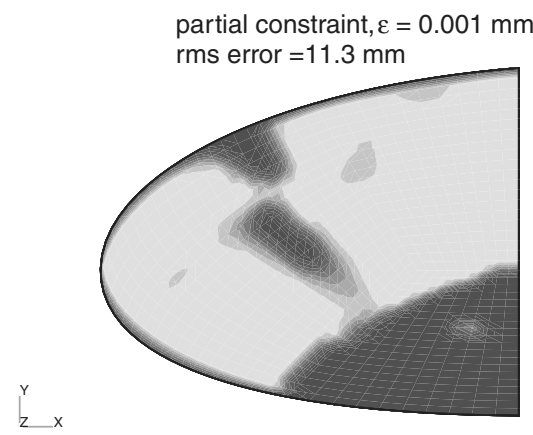

b)

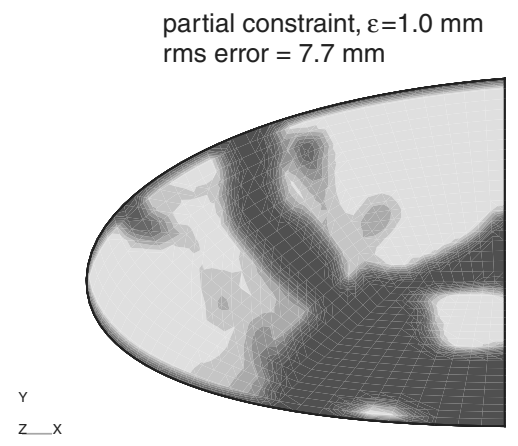

d)

Fig. 12 Density-based topology optimization solutions, illustrating the effects of shape-change constraints. 


\section{Conclusions}

In this paper the use of topology optimization to design an adaptive compliant aircraft wing leading edge has been presented. In particular, the load-path-based technique was modified to enable the required number of parameters to be substantially reduced. This was achieved by representing the starting lattice as an undirected network and determining the load paths by means of a KSSP algorithm. A complete knowledge of the load paths in a lattice enables the designer to parameterize only a subset containing the shortest paths up to a predefined limit. In this way the level of parametrization may be controlled. A solution to the dual-objective problem, requiring minimization of structural mass and rms shape-change error under a combination of internal actuation and a representation of the aerodynamic loading for a particular flight case, of the adaptive leading edge was achieved.

A disadvantage of the load-path-based method is that a parent lattice must be specified at the beginning of the process, requiring a subjective designer decision. The corresponding advantage, however, is that the resulting structure requires only limited postprocessing in order to be physically realized. An example of this postprocessing for the wing rib structure, requiring a reduction of the stress under deformation, was demonstrated, and a physical model was constructed. In a validation of the process, the model was observed to deform and reform under repeated actuation in a repeatable fashion.

As the load-path-based technique is not widely adopted it is of interest to compare the results achieved by means of the betterknown density-based topology optimization approach. Because of restrictions in the way the optimization problem may be defined in the commercially available implementation of the density-based method that was used, it was necessary to reformulate the shapechange objective as constraints. An investigation into how this reformulation should be carried out was performed. It was found that the density-based method did not reach solutions with shape-change errors as low as achieved with the load-path-based approach. A major advantage of the density-based approach, however, is that no designer subjectivity is required at the beginning of the design process; the design domain is fully populated with structure. Although the solutions do not fulfil the design objectives as well as those from the load-path-based approach, the topologies that are indicated, particularly the solution shown in Fig. $12 \mathrm{~b}$, are very close. Consequently, a density-based approach may be useful at the beginning of the design process to determine the appropriate topology, if not the geometry, in relatively few iterations. This will assist in the specification of the parent lattice in the load-path-based approach, removing a level of subjectivity.

The use of commercial software is beneficial as, despite restrictions in the optimization formulations, the option of a large number of additional loading types and elements means that the potential to use the process for design problems relevant to actual engineering applications is increased.

The load-based optimization presented in this paper may be extended in a number of ways through the incorporation of additional constraints and objectives. To reduce the required postprocessing, the maximum stress under actuation may be limited to the yield stress of the material. It may also be desirable to allow for different types of connections between members, such as fixed and hinged. This would enable the synthesis of structures with both concentrated and distributed compliance. Further constraints would be required to limit the presence of end hinges to members in tension, to avoid local buckling of living hinges. The feasibility constraint to avoid member crossover was implemented in the load-path-based analysis. This, however, could be extended to avoid the generation of members that are nearly parallel by constraining the minimum angle between adjacent members.

\section{Acknowledgments}

The work presented in this paper was funded under the Sixth Framework Programme of the European Commission. The authors would like to thank Ettore Baldassin, Aurelio Boscarino, and
Giovanni Carossa of Alenia-Aeronautica in Italy for their input into the design of the compliant wing. The assistance of Philippe Andry, Fréderic Cugnon, Christopher Morton, and Alain Remouchamps of Samtech in Belgium with the use and provision of SAMCEF, TOPOL and BOSS-quattro is gratefully acknowledged. In addition two anonymous reviewers are thanked for their detailed and helpful comments. This work was carried out at the University of Cambridge, where both authors were previously affiliated.

\section{References}

[1] Kota, S., Hetrick, J., Osborn, R., Paul, D., Pendleton, E., Flick, P., and Tilman, C., "Design and Application of Compliant Mechanisms for Morphing Aircraft Structures," Proceedings of SPIE: The International Society for Optical Engineering, Vol. 5054, No. 24, 2003, pp. 24-33. doi: $10.1117 / 12.483869$

[2] Kota, S., and Hetrick, J. A., "Adaptive Compliant Wing and Rotor System," FlexSys Inc., Patent No. WO/2004/108525, filed 16 Dec. 2004.

[3] Howell, L., Compliant Mechanisms,Wiley, New York, 2001.

[4] Lu, K.-J., and Kota, S., "An Effective Method of Synthesizing Compliant Adaptive Structures Using Load Path Representation," Journal of Intelligent Material Systems and Structures, Vol. 16, No. 4, 2004, pp. 307-317.doi:10.1177/1045389X05050104

[5] SAMCEF Finite Element Package, Ver. 12.0-03, Samtech Group, Belgium, 2007.

[6] BOSS-quattro, Ver. 6.0-01, Samtech Group Belgium, 2006.

[7] TOPOL Density-Based Topology Optimization Package, Samtech Group, Belgium, 2007.

[8] Lu, K.-J., and Kota, S., "Design of Compliant Mechanisms for Morphing Structural Shapes," Journal of Intelligent Material Systems and Structures, Vol. 14, No. 6, 2003, pp. 379-391. doi:10.1177/1045389X03035563

[9] Ponterosso, P., and Fox, D., "Going Organic: Using Evolution in Civils Design," Proceedings of the Institution of Civil Engineers, Vol. 160, No. 1, 2007, pp. 43-48. doi:10.1680/cien.2007.160.1.43

[10] Forrest, S., "Genetic Algorithms: Principles of Natural Selection Applied to Computation," Science, Vol. 261, No. 5123, 1993, pp. $872-878$.

doi: $10.1126 /$ science. 8346439

[11] Lu, K.-J., and Kota, S., "Topology and Dimensional Synthesis of Compliant Mechanisms Using Discrete Optimization," Journal of Mechanical Design, Vol. 128, No. 5, 2006, pp. 1080-1091. doi:10.1115/1.2216729

[12] Hoffman, W., and Pavley, R., "A Method for the Solution of the Nth Best Path Problem," Journal of the Association of Computing Machinery, Vol. 6, No. 4, 1959, pp. 506-514.

[13] Clarke, S., Krikorian, A., and Rausen, J., "Computing the $N$ Best Loopless Paths in a Network," SIAM Journal on Applied Mathematics, Vol. 11, No. 4, 1963, pp. 1096-1102. doi:10.1137/0111081

[14] Hadjiconstantinou, E., and Christofides, N., "An Efficient Implementation of an Algorithm for Finding $K$ Shortest Simple Paths," Networks, Vol. 34, No. 2, 1999, pp. 88-101. doi:10.1002/(SICI)1097-0037(199909)34:2\&lt;88::AIDNET2\&gt;3.0.CO;2-1

[15] Martins, E., and Pascoal, M., "A New Implementation of Yen's Ranking Loopless Paths Algorithm," 4OR-Quarterly Journal of the Belgian, French and Italian Operations Research Societies, Vol. 1, No. 2, 2003, pp. 121-134.doi:10.1007/s10288-002-0010-2

[16] SAMCEF, Ver 12.1 Documentation, Samtech Group, Belgium, 2007.

[17] Drela, M., "XFOIL: An Analysis and Design System for Low Reynolds Number Airfoils," Low Reynolds Number Aerodynamics, edited by T. J. Mueller, Vol. 54, Lecture Notes in Engineering, Springer-Verlag, New York, 1989.

[18] BOSS-quattro, Ver. 5 Documentation Samtech Group, Belgium, 2006.

[19] Santer, M., and Pellegrino, S., "Implementation of Load-Path Topology Optimisation in Samcef for the Generation of Compliant Structures," Proceedings of the 10th Samtech Users' Conference, Paper No. 36, 2007.

[20] Poncelet, F., Fleury, C., Remouchamps, A., and Grihon, S., "TOPOL: A Topological Optimization Tool for Industrial Design," Proceedings of the 6th World Congress of Structural and Multidisciplinary Optimization [CD-ROM], 2005, pp. 5141-5161.

[21] Bendsøe, M., and Sigmund, O., Topology Optimization: Theory, Methods, and Applications, Springer-Verlag, Berlin, 2nd ed., 2003.

[22] Kaza, R., Saikumar, S., and Wang, M., "Solid Free Form Design for 
Structural Optimization," Proceedings of the ESDA 2002: 6th Biennial Conference on Engineering Systems Design and Analysis, American Society of Mechanical Engineers, Paper ESDA2002/DES-010, 2002.

[23] Guest, J., Prevost, J., and Belytschko, T., "Achieving Minimum Length Scale in Topology Optimization Using Nodal Design Variables and Projection Functions," International Journal for Numerical Methods in Engineering, Vol. 61, No. 2, 2004, pp. 238-254.

doi:10.1002/nme.1064

[24] Sigmund, O., "Morphology-Based Black and White Filters for Topology Optimization," Structural and Multidisciplinary Optimization, Vol. 33, Nos. 4-5, 2007, pp. 401-424. doi:10.1007/s00158-006-0087-x
[25] Pedersen, C., Buhl, T., and Sigmund, O., "Topology Synthesis of Large-Displacement Compliant Mechanisms," International Journal for Numerical Methods in Engineering, Vol. 50, No. 12, 2001, pp. 2683-2705. doi: $10.1002 / \mathrm{nme} .148$

[26] Fleury, C., "CONLIN: An Efficient Dual Optimizer Based on Convex Approximation Concepts," Structural and Multidisciplinary Optimization, Vol. 1, No. 2, 1989, pp. 81-89.doi:10.1007/BF01637664

T. Zang Associate Editor 